\title{
Cutaneous Mucormycosis in a Trauma Patient with a New Diagnosis of Diabetes Mellitus
}

\author{
Georgina Alizo Arruebarrena, Andrea E. Romano, Jason Sciarretta, \\ Melanie Alesa Hopkins, and John Mihran Davis
}

\begin{abstract}
Background: Mucormycosis is an uncommon, but life-threatening fungal infection caused by the order $M u$ corales, family Mucoraceae. It is difficult to diagnose and manage, and it has a high fatality rate because of its rapid progression.

Methods: We present a 34-year-old male trauma patient admitted following a motor vehicle accident.

Results: While he had no medical history of diabetes mellitus, his admitting glycosylated hemoglobin (HbA1c) was $12.7 \%$. On hospital day eight the patient developed an ecchymotic lesion on his left flank associated with surrounding erythema and edema. The edges of the lesion became necrotic and surrounding erythema expanded on hospital day ten. A white cotton-like appearance formed over the ecchymotic wound. An extensive surgical debridement was performed, extending to healthy bleeding tissue. A skin biopsy showed broad, large and nonseparated fundal hyphae. On the same day, liposomal amphoteracin B at $5 \mathrm{mg} / \mathrm{kg} /$ day was started. The tissue culture was positive for Rhizopus species. The patient underwent multiple subsequent debridements and received a total course of 4 wks of liposomal amphoteracin B. Fifteen days from the initial surgical incision debridement, a split thickness skin grafting was performed.
\end{abstract}

Conclusion: No mucormycosis relapse was noted 3 mo after initial fungal infection presentation.

$\mathbf{M}$ UCORMYCOSIS IS AN UNCOMMON, but life-threatening fungal infection. It is almost exclusively observed in immunocompromised patients, including hematological malignancies, uncontrolled diabetics mellitus, or organ transplant patients. The infection is caused by the Mucorales fungal order, and it is classified as (1) rhinocerebral, (2) pulmonary, (3) gastrointestinal, (4) cutaneous, (5) disseminated, and (6) uncommon rare forms such as endocarditis, osteomyelitis, peritonitis, and renal infection [1], depending on the angioinvasion of a particular anatomic site and its clinical manifestations. These infections remain difficult to diagnose and manage. Furthermore, they have the potential to progress fairly rapidly, thus, an important index of suspicion in the high-risk populations is mandatory.

\section{Clinical History, Physical Exam}

A 34-year-old male unrestrained driver was admitted to the trauma service following a motor vehicle collision that resulted in a rollover of his vehicle. His initial injuries were a subarachnoid hemorrhage, skull fractures, facial fractures, a left radial fracture, and an open left tibial fracture with a popliteal artery injury. He was taken to the operating room emergently for his vascular injury. A left reverse saphenous vein interposition graft was used to repair the popliteal artery.

\section{Diagnosis, Intervention}

On hospital day two, a glycosylated hemoglobin level (HbA1c) was $12.7 \%$. Insulin sliding scale and long-acting insulin were used for glucose control. His preliminary ISS was 66. On hospital day seven, the patient was febrile up to 103.5. He also had associated leukocytosis. He was started on antibiotic therapy for Serratia pneumonia. On hospital day eight, the patient developed a new cutaneous lesion on his left flank. It was ecchymotic in nature, and associated with surrounding erythema and edema. At that time a two-centimeter incision was made in order to drain a suspected fluid collection without success. A computed tomography (CT) scan of his abdomen was taken and it showed only diffuse edema

(C) Georgina Alizo Arruebarrena et al. 2016; Published by Mary Ann Liebert, Inc. This Open Access article is distributed under the terms of the Creative Commons License (http://creativecommons.org/licenses/by/4.0), which permits unrestricted use, distribution, and reproduction in any medium, provided the original work is properly credited. 


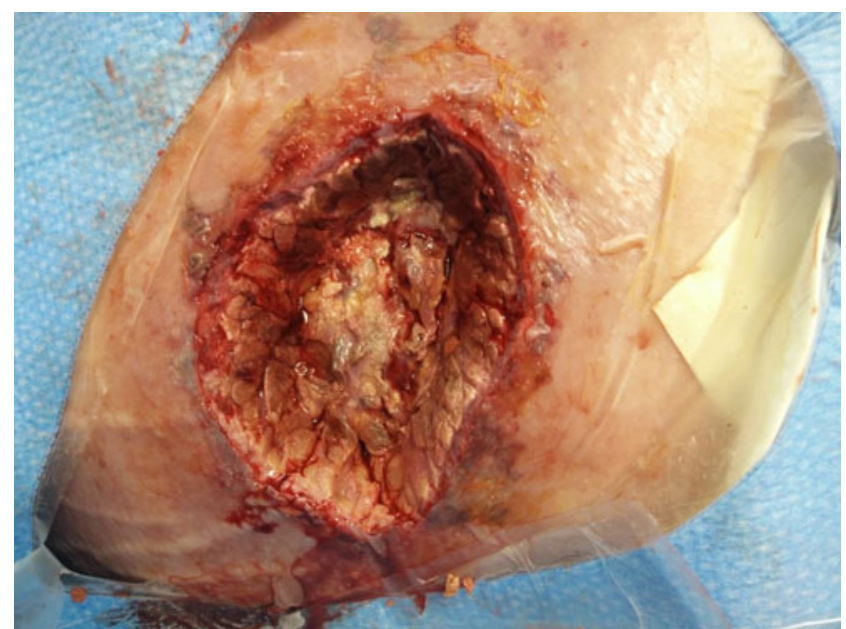

FIG. 1. Cutaneous mucormycosis on the flank of a trauma patient with undiagnosed diabetes mellitus.
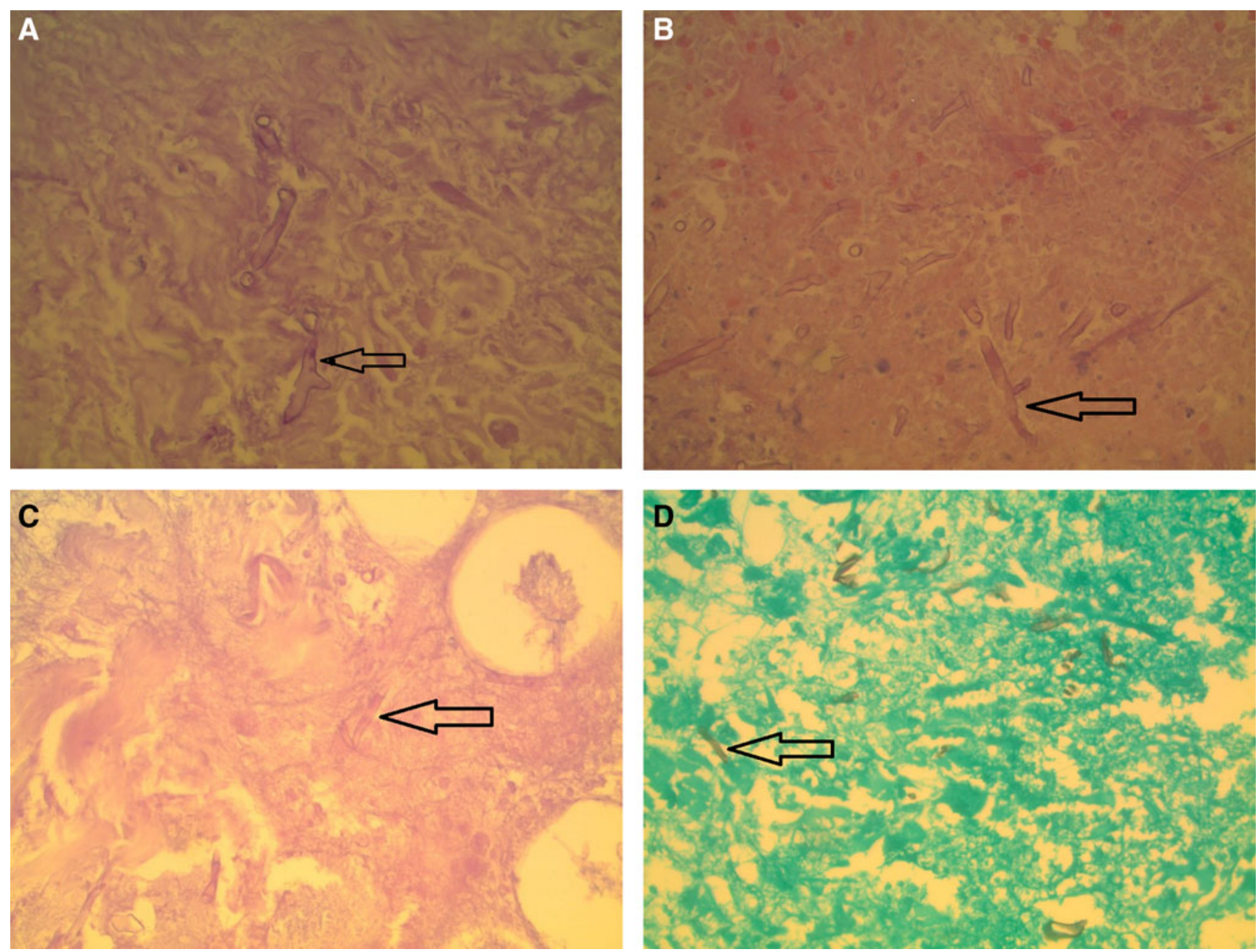

FIG. 3. Hematoxylin and eosin stain (A and B), periodic acid-Schiff stain $(\mathbf{C})$, and silver stain (D) showing cutaneous mucormycosis of the skin (arrows). Septa are not apparent and branches arise at right angles (1A). Hyphae are thin walled.

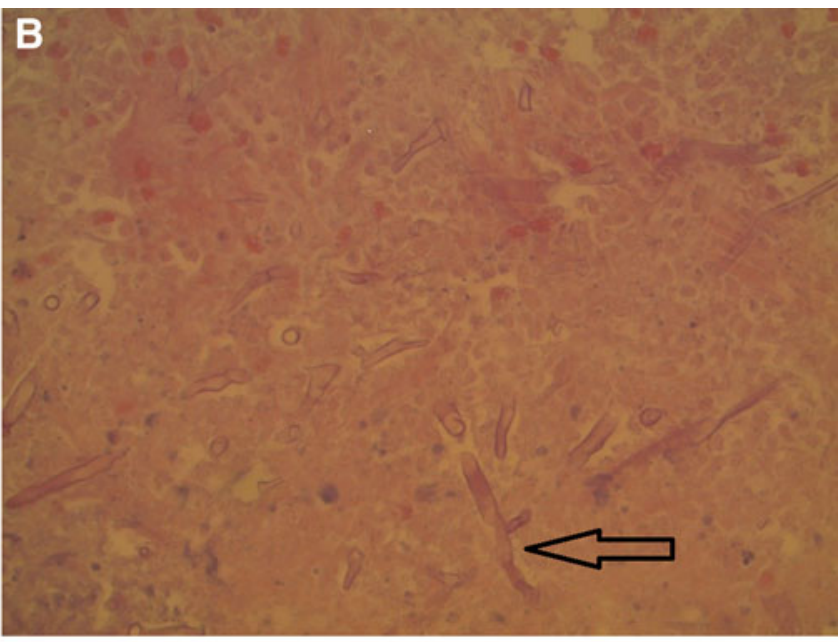

FIG. 2. Specimens from initial bedside debridement. The excised tissue has the appearance of a necrotizing infection. 


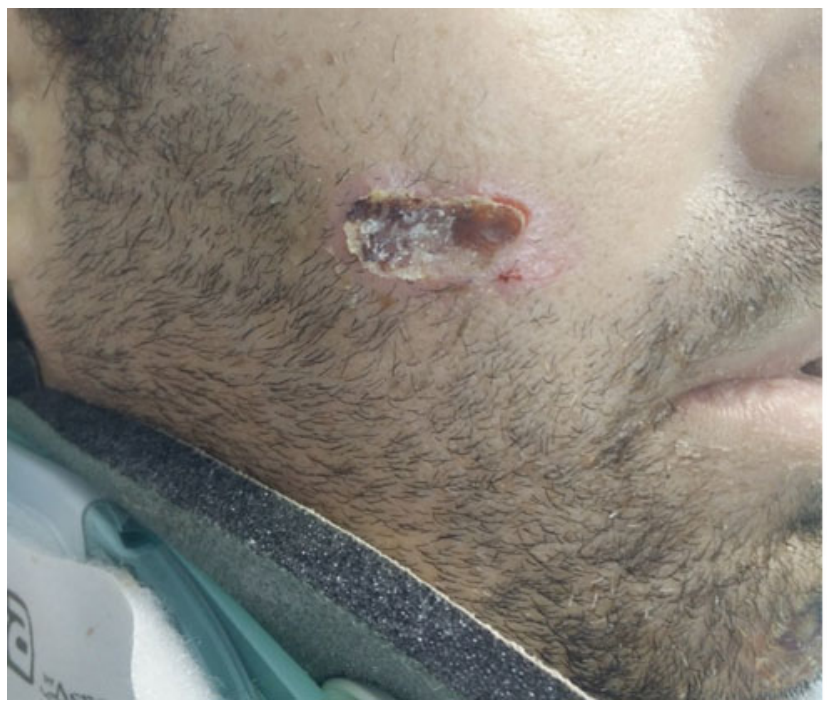

FIG. 4. Cotton-like appearance layer over facial scab.

in the left lower abdominal wall with no well-defined fluid collection.

On hospital day nine, the lesion evolved into a more pronounced ecchymosis. The diameter increased to $5 \times 5 \mathrm{~cm}$. On hospital day ten, the edges of the lesion became necrotic and surrounding erythema expanded (Fig. 1). A white cotton-like appearance over the ecchymotic portion was noted. A bedside debridement was performed to further evaluate the lesion, which was suspicious for a necrotizing soft tissue infection. The need for a larger debridement was established. The patient was taken to the operating room for a more extensive debridement (Fig. 2). The entirety of the non-viable tissue was excised and healthy bleeding tissue was seen for a final $15 \times 15 \mathrm{~cm}$ defect.

On the same day, antibiotic therapy was broadened. Liposomal amphoteracin B at $5 \mathrm{mg} / \mathrm{kg} /$ day was also started because of a high suspicion of fungal infection. A skin biopsy was sent for cultures, with smear and tissue sample showing broad, large, and non-separated fundal hyphae. Histopathological findings revealed that the organism was growing in the surgically removed tissue (Fig. 3). The tissue culture was positive for Rhizopus species.

On hospital day 12, a new white cotton-like appearance layer over facial scabs were observed on the patient forehead and cheeks (Fig. 4). These lesions never progressed into necrotizing cutaneous lesions. On the following days, the patient underwent beside debridement of small amounts of non-viable tissue every other day for the next six days, debridement becoming less significant and less frequent. The patient received a total course of 4 wk of liposomal amphoteracin B, and on post-operative day 15 , from initial debridement, a split thickness skin grafting was successfully performed. No mucormycosis relapse was noted 3 mo after initial fungal infection presentation.

\section{Discussion}

Diabetes mellitus is a well-known risk factor for mucormycosis infections with episodes of diabetic ketoacidosis considered especially to put patients at risk [2]. In a recent review [1], mucormycosis was found to be the diabetes mellitus-defining illness in $16 \%$ of a population composed of 337 diabetics.

Mucorales are typically found in the soil and are associated with decaying organic matter, such as leaves, compost piles, or rotten wood [3]. Open wounds are usually the point of entrance of these fungi. They are associated with angioinvasive disease, often leading to thrombosis, infarction of involved tissues, and tissue destruction mediated by a number of fungal proteases, lipases, and mycotoxins. This angioinvasion is associated with the ability of the organism to hematogenously disseminate from the original site of infection to other target organs.

Some believe consequences of cutaneous manifestation of mucormycosis may be underestimated. In a review [4], $20 \%$ of patients with cutaneous disease developed hematogenous dissemination from the original cutaneous site to another organ, resulting in an overall mortality of $94 \%$ in this subgroup. On the other hand, out of 220 cases of hematogenously disseminated infection, only six had documented cutaneous involvement. They also compared treatment options and outcomes. Of the 929 cases reviewed, 64\% received only some form of antifungal chemotherapy, with a survival rate $62 \%$ on this subgroup. Of these 596 patients on this sub-group, 532 $(89 \%)$ received amphotericin B deoxycholate, with an overall survival of $61 \%$. Surgical management alone was received by $9.68 \%$ of patients with a survival rate of $57 \%$. Those who received a combination of surgery and anti-fungal chemotherapy saw an increased in survival rate to $70 \%$.

Our patient received the combination of surgical and medical management upon diagnosis of the infection. Having a high index of suspicion of fungal infection in this severely ill patient who was recently diagnosed with diabetes mellitus helped in the decision making, and guided the aggressive management of the infection and subsequent successful treatment. In conclusion, a prompt diagnosis, an extended surgical resection, and an anti-fungal chemotherapy are essential components required for effective treatment of mucormycosis and increased survival. Adjustment of modifiable risk factors is also crucial in order to obtain an adequate control of cutaneous mucormycosis. In our case, tight glycemic control was absolutely necessary, and it was achieved.

\section{Author Disclosure Statement}

No competing financial interests exist.

\section{References}

1. Petrikkos G, Skiada A, Lortholary O, et al. Epidemiology and clinical manifestations of mucormycosis. Clin Infect Dis 2012;54Suppl:S23-S34.

2. U.S. Centers for Disease Control and Prevention. National diabetes surveillance system: Diabetic ketoacidosis as firstlisted diagnosis. www.cdc.gov/diabetes/statistics/dkafirst. (Last accessed December 30, 2015.)

3. www.cdc.gov/fungal/diseases/mucormycosis. (Last accessed December 30, 2015.) 
4. Roden MM, Zaoutis TE, Buchanan WL, et al. Epidemiology and outcome of mucormycosis: A review of 929 reported cases. Clin Infect Dis 2005;41:634-653.

Address correspondence to: Dr. John Mihran Davis Department of Clinical Surgery University of South Carolina Department of Surgery Grand Strand Medical Center 809 82nd Parkway Myrtle Beach, SC 29572

E-mail: john.davis2@hcahealthcare.com

\begin{tabular}{|c|}
$\begin{array}{c}\text { Abbreviation Used } \\
\mathrm{CT}=\text { computed tomography }\end{array}$ \\
\hline
\end{tabular}

Cite this article as: Alizo Arruebarrena G, Romano AE, Sciarretta J, Alesa Hopkins M, Mihran Davis J (2016) Cutaneous mucormycosis in a trauma patient with a new diagnosis of diabetes mellitus, Surgical Infections Case Reports 1:1, 4-7, DOI: 10.1089/crsi.2016.29001.gaa. 